\title{
RELATIONSHIP BETWEEN STIGMA AND MENTAL HEALTH OF PHYSICALY DISABLED: MEDIATING EFFECT OF RESILIENCE
}

\author{
Lei Liu ${ }^{1} \&$ Yu Zhang ${ }^{2}$ \\ ${ }^{1}$ School of Economics, Wuhan Textile University, Wuhan, China \\ ${ }^{2}$ School of Economics \& Management, Hubei University of Arts and Science, Xiangyang, China
}

received: 21.4.2021;

revised: 8.8.2021;

accepted: 23.10 .2021

\section{SUMMARY}

Background: The physically disabled is easy to develop a psychological problem due to long-term limitation of motion and inconvenience in social communication. There's still no consensus on whether stigma is related to the mental health of the physically disabled. However, resilience is closely related to mental health. To determine the influencing mechanism of stigma and resilience on mental health, the influences of stigma on anxiety and depression as well as the role of resilience in this process were explored in this study.

Subjects and methods: A total of 200 physically disabled people who had needs of rehabilitation were chosen from 5 communities surrounding Wuhan University of Technology from April 2020 to October 2021. Subjects' resilience, stigma, and mental health level were investigated by the Connor-Davidson resilience scale (CD-RISC), social impact scale (SIS), self-rating depression scale (PHQ-9), and Generalized Anxiety Disorder (GAD-7).

Results: Stigma of the physically disabled is positively related to anxiety and depression $(r=0.618,0.642, P<0.05)$, and it is negatively related to resilience $(r=-0.561)$. Anxiety and depression are negatively related to resilience $(r=-0.673,-0.665, P<0.05)$. Resilience can partially mediate the relationship between stigma and anxiety and depression. The mediating effect values are 0.10 and 0.18 , respectively.

Conclusions: The stigma of the physically disabled is closely related to resilience, anxiety, and depression. It not only can influence mental health both directly and indirectly through the mediating effect of resilience.

Key words: physically disabled - resilience - stigma - anxiety - depression

\section{INTRODUCTION}

The disabled refers to the people who have lost some tissues, functions or abnormal physiological and body structure, partially or completely lost the ability to engage in activities in the normal way. According to the results of the second national sampling survey of the disabled in China, the disabled population reached as high as 82.96 million, accounting for $6.34 \%$ of the total population in China (Zheng et al. 2019). Physical disability refers to different degrees of functional and structural damages in the motor system of the human body caused by limb deformity or body paralysis and deformity and the physically disabled population accounts for $29.07 \%$ of the total disabled population (Furlan et al. 2018). The physically disabled is easy to develop a psychological problem due to long-term limitation of motion and inconvenience in social communication, which is mainly manifested by strong sense of inferiority and anxiety, depression, and even psychological crisis (Chen et al. 2019). Physical pains interact with mental and psychological factors, which can cause cognitive, emotional, and behavioral disorders of the physically disabled, thus causing adverse impacts on daily life, social communication state, and quality of life (Debicka et al. 2020). Which factors are related to the psychological problems of the physically disabled? Whether it is possible to recognize individuals with psychological problems through these factors and adopt effective intervening measures timely is of important significance to improve the mental health of the physically disabled.

Stigma refers to the inner experiences that individuals feel shamed for having a disease. It is originated from the prejudice and isolation of the social public to patients due to the stereotype image as well as selfdiscrimination and self-abasement of patients after internalization. Currently, researches on stigma mainly concentrate on the discussion on internal structures of stigma. The involved fields mainly concentrated on stigma, mental health, individual response, and expectation level of individuals. Most studies are about the relationship between stigma and mental health. Lei et al. (2018) to study the status of stigma and coping styles in patients with prostatic hyperplasia and explore reasonable and effective nursing intervention measures, they investigated one hundred and fifty-one patients with prostatic hyperplasia by general data questionnaire, social impact scale (SIS), and simple coping style questionnaire (SCSQ). They found that inner shame and social isolation were the highest with strong stigma. There were differences in stigma among patients with different education levels, payment methods of medical expenses, disease-related knowledge, and severity of symptoms. Compared with the domestic model, negative coping scores were significantly higher, and 
positive coping scores were significantly lower. There was a positive correlation between negative coping style and total score of stigma and score of all dimensions, and positive coping style was negatively correlated with total score of stigma, internal shame dimension and social isolation dimension. At last, they concluded that there is a strong sense of stigma in patients with prostatic hyperplasia, and it is relatively passive about the disease. The nursing staff should help them alleviate the stigma by improving patients' coping style, enhance their confidence in coping with diseases, and improve patients' quality of life. Chen et al. (2020) investigated the correlation between the level of stigma and psychological resilience of patients after maxillofacial surgery and its influencing factors. They used a convenient sampling method to select 80 patients who underwent maxillofacial surgery for cleft lip and palate, maxillofacial tumors, trauma, etc. from 3 hospitals in Shandong and Jilin provinces from June to December 2018, using General Questionnaires, Social Impact Scale (SIS), and used Connor-Davidson Resilience Scale (CD-RISC) as research tools to investigate patients. They found that the SIS score of 80 patients undergoing maxillofacial surgery was $(64.75 \pm 12.52)$, and the CD-RISC score was $(26.37 \pm 7.06)$. The results of correlation analysis showed that the level of patients' stigma was negatively correlated with psychological resilience. The results of multiple linear regression analysis showed that gender, age, education level, postoperative time, recurrence and number of operations and level of mental resilience were the influencing factors of patients' level of stigma. Then they concluded that patients with maxillofacial surgery have a relatively high level of stigma and are jointly restricted by various factors. Nursing staff should adopt active psychological intervention strategies according to the different conditions of the patients, improve the patients' psychological resilience level, and effectively reduce the patients' sense of stigma. Sun et al. (2018) found that stigma is common in patients with breast cancer which can result in social isolation, medical delay, quality of life decline and many other issues. And they summarized the research status and intervention measures of stigma in patients with breast cancer, aiming to provide a theoretical basis for the effective intervention measures. According to data, stigma is closely related to the mental health level of individuals. Decreasing stigma is conducive to improving the hope and self-esteem level of patients and relieving their negative emotions. Stigma is a factor that can influence mental health (Chen et al. 2020, Hernandez et al., 2016). Some studies pointed out that stigma is positively related to anxiety and depression, but it is negatively related to activity of daily living. A hypothesis can be proposed that stigma might be an antecedent variable that influences mental health (Du et al. 2018).

Additionally, resilience refers to the good response and adaptation of individuals to setbacks and it can regulate the relationship between negative life events and mental health. People with stronger resilience have better mental health quality. Chang et al. (2019) thought that the mental health profession exposes healthcare workers to unique stressors such as associative stigma (stigmatization that is extended from the stigmatized patients to psychiatric professionals and is based on affiliation with an individual with mental illness). Enhancing resilience, or the ability to 'bounce back' from adversity, is found to be useful in reducing occupational stress and its negative effects. Given the high burnout rates reported among mental health professionals, they tried to examine resilience in this group of professionals and to explore the association between resilience and associative stigma. Then they used the Brief Resilience Scale (BRS) and made participants complete questionnaires that examined associative stigma. They found that associative stigma remained significantly associated with resilience score after controlling for sociodemographic factors whereby higher associative stigma predicted lower resilience scores. So they pointed out that the present finding suggests that resilience-building programmers among mental health workers should target those of the younger age group and that addressing the issue of associative stigma is essential. Wu et al. (2020) explored the correlation between social support, psychological resilience and coping style of the caregivers of stroke patients. She selected the caregivers of 209 stroke patients hospitalized in her hospital as the study objects, and used the social support assessment scale, psychological resilience scale and coping style scale for analyzed the correlation between social support and psychological resilience, coping styles. She found that the subjective support was positively correlated with tenacity, self -improvement, psychological resilience, and positive coping, and negatively correlated with negative coping, the objective support was not significantly correlated with the psychological resilience of the subjects and coping styles, the support utilization was positively correlated with positive coping and negatively correlated with negative coping. The social support scores had positively correlated with tenacity, psychological resilience scores, and positive coping, and had a significant negative correlation with negative coping. At last she concluded that the social support of the caregivers in stroke has a certain correlation with psychological resilience and coping styles and that the caregivers with high social support have higher psychological resilience and are more inclined to implement positive coping styles. Li et al. (2020) evaluated the relationship between problem-solving, coping styles, and clinical nurses' resilience. They investigated 1073 clinical nurses with general information questionnaire, the Connor-Davidson Resilience Scale, the Chinese Version of the Social Problem-Solving Inventory, Simplified Coping Style Questionnaire. They found that the score of the resilience of clinical nurses was at a moderate level. 
Positive coping style, negative problem orientation, avoidance style, impulse/neglect style were the main influencing factors of resilience. Positive coping became a major predictor of protective factors for resilience, and the negative problem orientation became the main predictor of risk factors for resilience. Analysis of interaction effect showed that positive coping and problem-solving abilities had positive effects on resilience, the negative problem orientation, avoidance style and impulse/neglect style had negative effects on resilience. Problem-solving ability played a partial mediating role between resilience and positive coping. So they thought that nursing administrators should pay attention to the resilience of clinical nurses and take measures to carry out relevant training and education, enhance the resilience level of nurses to prevent the psychological crisis. Low-level resilience may influence adjustment individuals' responses to negative stress, thus triggering negative emotions of anxiety and depression ( $\mathrm{Li}$ et al. 2019). According to existing studies, good resilience plays an important role in improving the mental health level and subjective happiness of different groups (Arabaci et al. 2018, Yin et al. 2019). Therefore, it can be further deduced that the stigma of the physically disabled may not influence mental health directly. Instead, resilience might play a mediating effect between stigma and mental health. However, such indirect influencing mechanism remains unknown in existing studies.

To sum up, this study explored the relationship between stigma, resilience, and mental health as well as the mediating effect of resilience between stigma and mental health. A total of 220 respondents with physical disabilities were chosen to investigate their stigma, resilience and mental health.

\section{SUBJECTS AND METHODS}

\section{Samples Selection}

A total of 220 respondents with physical disabilities and needed rehabilitation were chosen from 5 communities surrounding Wuhan University of Technology from April 2020 to October 2021 as the research objects.

Inclusion criteria:

- Age $\geq 18$;

- Basic communication and expression ability;

- Being able to finish scales;

- Signing the informed consent.

Exclusion criteria:

- Patients with serious consciousness, intelligence, and mental disorders;

- Patients who are unwilling to cooperate or communicate;

- Patients with history of drug abuse;

- Patients with data missing.

\section{Measuring Tools}

\section{Connor-Davidson resilience scale (CD-RISC)}

This scale covers 20 items of three dimensions of optimism, strength and tenacity, including 4 items of optimism, 3 items of strength and 13 items of tenacity (Ong et al. 2018). Each item used the 5-level scoring method: never: 0; hardly: 1; sometimes: 2 ; often: 3 ; always: 4 . The total score of the scale is $0-100$ scores and the lower score indicates the lower resilience. The coefficient of internal consistency of CD-RISC is 0.91 , indicating the good reliability and validity of the scale.

\section{Social impact scale (SIS)}

This scale covers 24 items of four dimensions, including social isolation (7 items), economic discrimination (3 items), social exclusion (9 items) and intrinsic shame (5 items) (Yuan et al. 2018). Each item used the 4-level scoring method: strongly disagree: 1 ; disagree: 2 ; agree: 3; strongly agree: 4 . The total score of the scale is $24-96$ scores and the lower score indicates the lower stigma level. Low stigma level: $1 \leq$ average score of items $<2$; moderate stigma: $2<$ average score of items $<3$; high stigma level: 3 -average score of items. The coefficient of internal consistency of CD-RISC is 0.85 , indicating the good reliability and validity of the scale.

\section{Self-rating depression scale (PHQ-9)}

This scale is composed 9 dimensions, including low self-evaluation, eating disorder, lack of energy, sleep disorder, depression, loss of happiness, passivity, difficulties in concentration and slow to act (Tomitaka et al. 2018). Each dimension is evaluated from 0-3 scores, and the total score ranges from 0 to 27 . A higher score indicates a more serious depression. No depression: $0-4$; mild: $5-9$; moderate: $10-14$; severe: $\geq 15$. The coefficient of internal consistency of CD-RISC is 0.93 , indicating the good reliability and validity of the scale.

\section{Generalized Anxiety Disorder (GAD-7)}

This scale is composed of 7 dimensions, such as difficulties in relaxing, lack of interest or happiness in doing things, excessive concern to various problems, etc (Esser et al. 2018). Each dimension is evaluated from 03 scores, and the total score ranges 0-21. A higher score indicates more serious anxiety. Normal: $<5$; mild: $5 \leq$ scores $<10$; moderate: $10 \leq$ scores $<15$; severe: $\geq 15$. The coefficient of internal consistency of CD-RISC is 0.96 , indicating the good reliability and validity of the scale.

\section{Statistical Analysis}

The common method bias was tested by factor analysis based on SPSS 22.0. Correlation coefficients of variables were investigated by Pearson correlation analysis. The mediating effect of resilience between stigma and mental health of the physically disabled was tested by Hayes's Process. 


\section{RESULTS}

\section{Common method bias test}

The common method bias effect was tested by Harman single-factor test method. Results demonstrate that there are 12 factors with eigenvalue higher than 1 . The amount of variation explained by the first factor is $28.83 \%$, which is lower than the critical standard of $40 \%$. Therefore, this study has no obvious common method bias.

\section{Correlation analysis among stigma, mental health, and resilience of the physically disabled}

According to correlation analysis, the total scores of stigma and scores of different dimensions of the physically disabled show significantly positive correlations with depression and anxiety $(P<0.05)$. The total scores of resilience and scores of different dimensions show significantly negative correlations with stigma, depression, and anxiety $(P<0.05)$. Results are shown in Table 1 and Table 2.

\section{Mediating effect of resilience between stigma and mental health of the physically disabled}

All continuous variables were normalized. Depression was chosen as a dependent variable, and stigma was used as an independent variable, while resilience was used as the mediating variable to test its mediating effect. Under control over gender, age, education, disability degree and marriage, stigma has a significantly positive main effect on depression of the physically disabled $(\beta=0.46, P<0.001)$, and resilience has a significantly negative main effect on depression $(\beta=-0.42$, $P<0.001)$. Anxiety was chosen as a dependent variable and stigma was used as an independent variable, while resilience was used as the mediating variable to test its mediating effect. Under control over gender, age, education, disability degree and marriage, stigma has a significantly positive main effect on the anxiety of the physically disabled $(\beta=0.50, P<0.001)$ and significantly negative main effect on resilience $(\beta=-0.59, P<0.001)$, and resilience has a significantly negative main effect on anxiety $(\beta=-0.46, P<0.001)$. Resilience provides a partially mediating effect between stigma and mental health of the physically disabled. Results are shown in Table 3.

Table 1. Mean and standard deviation of variables

\begin{tabular}{lrc}
\hline Variable & Average & Stand. Deviation \\
\hline Stigma & 8.561 & 2.314 \\
Social Isolation & 11.964 & 3.512 \\
Economic Discrimination & 13.018 & 4.021 \\
Social Exclusion & 10.899 & 3.027 \\
Intrinsic Shame & 16.524 & 4.351 \\
Psychological Resilience & 20.684 & 3.483 \\
Optimism & 26.145 & 5.361 \\
Strength & 30.869 & 4.875 \\
Tenacity & 24.815 & 5.027 \\
Depression & 21.434 & 6.124 \\
Anxiety & 4.531 & 1.244 \\
\hline
\end{tabular}

Table 2. Correlation analysis of stigma, mental health and resilience of the physically disabled

\begin{tabular}{|c|c|c|c|c|c|c|c|c|c|c|c|}
\hline Variable & 1 & 2 & 3 & 4 & 5 & 6 & 7 & 8 & 9 & 10 & 11 \\
\hline Stigma & - & & & & & & & & & & \\
\hline Social Isolation & $0.951^{* * *}$ & - & & & & & & & & & \\
\hline Economic Discrimination & $0.894^{* * *}$ & $0.712^{* * *}$ & - & & & & & & & & \\
\hline Social Exclusion & $0.923^{* * *}$ & $0.679^{* * *}$ & $0.653^{* * *}$ & - & & & & & & & \\
\hline Intrinsic Shame & $0.872^{* * *}$ & $0.694^{* * *}$ & $0.651^{* * *}$ & $0.674^{* * *}$ & - & & & & & & \\
\hline Psychological Resilience & $-0.561^{* * *}$ & $-0.531^{* * *}$ & $-0.543^{* * *}$ & $-0.527^{* * *}$ & $-0.509^{* * *}$ & - & & & & & \\
\hline Optimism & $-0.524^{* * *}$ & $-0.498^{* * *}$ & $-0.516^{* * *}$ & $-0.505^{* * *}$ & $-0.486^{* * *}$ & $0.931^{* * *}$ & - & & & & \\
\hline Strength & $-0.547^{* * *}$ & $-0.503^{* * *}$ & $-0.523^{* * *}$ & $-0.492^{* * *}$ & $-0.473^{* * *}$ & $0.962^{* * *}$ & $0.762^{* * *}$ & - & & & \\
\hline Tenacity & $-0.518^{* * *}$ & $-0.475^{* * *}$ & $-0.486^{* * *}$ & $-0.467^{* * *}$ & $-0.471^{* * *}$ & $0.918^{* * *}$ & $0.714^{* * *}$ & $0.736^{* * *}$ & - & & \\
\hline Depression & $0.642^{* * *}$ & $0.581^{* * *}$ & $0.611^{* * *}$ & $0.590^{* * *}$ & $0.554^{* * *}$ & $-0.665^{* * *}$ & $-0.568^{* * *}$ & $-0.524^{* * *}$ & $-0.541^{* * *}$ & - & \\
\hline Anxiety & $0.618^{* * *}$ & $0.563^{* * *}$ & $0.603^{* * *}$ & $0.575^{* * *}$ & $0.588^{* * *}$ & $-0.673^{* * *}$ & $-0.517^{* * *}$ & $-0.567^{* * *}$ & $-0.518^{* * *}$ & $0.682^{* * *}$ & - \\
\hline
\end{tabular}

Table 3. Mediating effect of resilience between stigma and mental health

\begin{tabular}{cccc}
\hline Path & $\beta$ & SE & 95\%CI \\
\hline Stigma $\rightarrow$ Psychological Resilience $\rightarrow$ Depression & & & {$[0.31 \sim 0.49]$} \\
Direct Effect & 0.46 & 0.07 & {$[0.12 \sim 0.23]$} \\
Indirect Effect & 0.18 & 0.06 & {$[0.35 \sim 0.51]$} \\
Stigma $\rightarrow$ Psychological Resilience $\rightarrow$ Anxiety & & & 0.05 \\
Direct Effect & 0.50 & 0.03 & {$[0.14 \sim 0.26]$} \\
Indirect Effect & 0.10 &
\end{tabular}




\section{DISCUSSION}

It can be seen from Table 1 and Table 2 that stigma, anxiety and depression and resilience of the physically disabled are closely related. The physically disabled with the higher stigma have the more serious depression and anxiety, and the lower resilience level. This agrees with previous research results (Kilic et al. 2013, Yan \& Zhang 2018). This might be because due to different appearances, the physically disabled is easy to suffer impaired self-esteem, self-undervalue, high loneliness and unwillingness of social communication, thus resulting in social isolation. Due to the missing of some body parts or limbs, the physically disabled become disability and they cannot recognize their values by working, thus having different incomes from normal people. Most of the physically disabled are in poverty and they are easy to suffer economic discrimination. Sullivan et al. (2016) also pointed out stigma is a cause of anxiety and depression in patients.

During daily life and social communication, there's a stereotyped image to appear and the public is easy to judges people with their appearance. As a result, the physically disabled are more sensitive to other people's attitudes. They will avoid communication with the external world during learning and life, thus increasing the internal stigma. Moreover, the physically disabled are discriminated against for physical anomaly or missing and are treated unfairly. If they live in such social environment for a long period, they become more nervous than physically normal people and they are easy to have psychological problems. Besides, some of the acquired physically disabled have stable career and family conditions before the trauma, but they fail to fulfill the original family and social role after. Such great sense miscues are also very easy to cause psychological problems.

It can be seen from Table 3 that resilience provides some mediating effect between stigma and mental health of the physically disabled. This demonstrates that stigma can influence the mental health of the physically disabled directly and directly through resilience. The physically disabled with relatively low resilience levels often care about others' attitudes (including family and society) to their physical disability too much. They avoid communicating with others, which finally leads to isolating themselves from the family and society. As a result, stigma is aggravated. Resilience has positive effect on the physically disabled. It is an important protective factor that can improve the optimism and tenacity of the physically disabled in adversity so that the physically disabled can adapt to attitudes of the society toward them and adopt more positive responses (Alschuler et al. 2016, Sharpley et al. 2016). Ge et al. (2018) found by analyzing mental health conditions of soldiers that resilience provides some mediating effect between anxiety sensitivity and mental health. They pointed out that it is necessary to improve psychological resilience to improve their mental health. This also fully demonstrates the important significance of resilience to mental health. Facing with bad life events, individuals who have good resilience are more willing to accept different appearances and influences on daily life more optimistically and positively, and they are willing to try to solve these bad influences (Jakovljevic 2018, Mandić \& Pavlović 2020), improve the quality of life through their efforts, and adapt to physical changes in a short period. Therefore, they have relatively lower stigma levels, milder anxiety and depression, and better mental health.

\section{CONCLUSION}

The physically disabled have a relatively high stigma, anxiety, and depression, as well as relatively poor resilience. Stigma is positively related to anxiety and depression, while anxiety and depression are negatively related to resilience. Stigma is negatively related to resilience. Resilience provides some mediating effect between stigma and anxiety and depression.

\section{Acknowledgements: None.}

\section{Conflict of interest: None to declare.}

\section{Contribution of individual authors:}

Lei Liu: Conception and design, analysis, planning and designing data collection

Yu Zhang: Material preparation, statistical analysis, Draft preparation, approval of the final version.

All authors have read and agreed to the published version of the manuscript.

\section{References}

1. Alschuler KN, Kratz AL, Ehde DM: Resilience and vulnerability in individuals with chronic pain and physical disability. Rehabil Psychol 2016; 61:7-18

2. Arabaci LB, Dikec G, Buyukbayram A, Uzunoglu G, Ozan E: Traumatic growth and psychological resilience status of female victims of violence inpatients in a district psychiatric hospital. Archives of psychiatric nursing 2018; 32:568-573

3. Chang S, Picco L, Abdin E, Yuan Q, Chong SA, Subramaniam M: Resilience and associative stigma among psychological health professionals in a tertiary psychiatric hospital: a cross-sectional study in Singapore. BMJ Open 2019; 9:e033762

4. Chen HG, Huang YQ, Liu ZR, Ma C, Zhang TT: Comorbidity and related factors of mental disability in physically disabled persons in China. Chinese Mental Health Journal 2019; 33:571-576

5. Chen L, Ma MS, Song HY, Yang M, Wang W, Zhang TX: Study on the correlation between stigma and anxiety in hospitalized patients with coronavirus disease 2019. Chinese Journal of Nursing 2020; 55:601-602

6. Chen X, Liu DL, Wang XL, Zhang Y: Influencing factors of stigma in patients with maxillofacial surgery. Chinese Journal of Modern Nursing 2020; 26:1758-1763 
7. Debicka J, Mazurek E, Szalonka K: Health Status and Lifestyle of the Mentally and Physically Disable. European Research Studies Journal 2020; 23:488-499

8. Du J, Chen YQ, Wu L, Duan Y: The reliability and validity of the Chinese version of the stigma scale for chronic illness 8-items in patients with neurological disorders. Journal of North Sichuan Medical College 2018; 33:301-304

9. Esser P, Hartung TJ, Friedrich M, et al: The Generalized Anxiety Disorder Screener (GAD-7) and the anxiety module of the Hospital and Depression Scale (HADS-A) as screening tools for generalized anxiety disorder among cancer patients. Psychooncology 2018; 27:1509-1516

10. Furlan AD, Irvin E, Munhall C, et al: Rehabilitation service models for people with physical and/or mental disability living in low- and middle-income countries: A systematic review. Journal of Rehabilitation Medicine 2018; 50:487-498

11. Ge YN, Ma KX, Cai WP, Zhang SM, Tong W: Relationship between anxiety sensitivity and mental health of soldiers: mediating effect of resilience. Academic Journal of Second Military Medical University 2018; 39:92-96

12. Hernandez SH, Morgan BJ, Parshall MB: Resilience, stress, stigma, and barriers to mental healthcare in U.S. air force nursing personnel. Nursing Research 2016; $65: 481-486$

13. Jakovljevic M: Empathy, Sense of Coherence and Resilience: Bridging Personal, Public and Global Mental Health and Conceptual Synthesis. Psychiatr Danub 2018; 30:380-384

14. Kilic SA, Dorstyn DS, Guiver NG: Examining factors that contribute to the process of resilience following spinal cord injury. Spinal Cord 2013; 51:553-557

15. Lei H, Li LS, Yang M, Zhang J: Correlation between stigma and coping styles in patients with prostatic hyperplasia. Modern Clinical Nursing 2018; 17:7-11

16. Li YM, Huang Y, Huang Y, Sun JH: Study on the relationship among resilience, problem solving and coping styles of clinical nurses. Journal of Nursing Administration 2020; 20:328-333

17. Li YZ, Zhang YP, Xiong M: The resilience characteristics and its influencing factors in the elderly disabled. Chinese Journal of Behavioral Medicine and Brain Science 2019; 28:354-358
18. Mandić $K \&$ Pavlović $K$ : Resilience in health and illness. Psychiatr Danub 2020; 32:226-232

19. Ong HL, Vaingankar JA, Abdin E, et al: Resilience and burden in caregivers of older adults: moderating and mediating effects of perceived social support. BMC Psychiatry 2018; 18:27

20. Sharpley $C F$, Bitsika V, Jesulola E, Fitzpatrick $K$ : The association between aspects of psychological resilience and subtypes of depression: implications for focused clinical treatment models. International Journal of Psychiatry in Clinical Practice 2016; 20:1-6

21. Sullivan KD, Kropp CM, Mofield B, Delgaty L, Contardo $C$ : The relationship between stigma and self-reported symptoms of anxiety and depression in Parkinson's disease. Clinical Neuropsychologist 2016; 25:212-216

22. Sun WJ, Chen DD, Wang J, Liu JQ, Zhang W: Research progress of stigma in patients with breast cancer. Chinese Nursing Management 2018; 18:692-695

23. Tomitaka S, Kawasaki Y, Ide K, Akutagawa M, Yamada H, Ono Y, Furukawa TA: Distributional patterns of item responses and total scores on the PHQ-9 in the general population: data from the National Health and Nutrition Examination Survey. BMC Psychiatry 2018; 18:108

24. Wu XY: Correlation analysis of social support, psychological resilience and coping style of the caregivers of stroke patients. Nursing Practice and Research 2020; 17:30-32

25. Yan H, Zhang X: Psychological resilience of patients with permanent stoma of rectal cancer and its correlation with stigma and psychological pain. International Journal of Pathology and Clinical Medicine 2018; 38:1027-1031

26. Yin ZX, Brasher MS, Kraus VB, Lv YB, Shi XM, Zeng Y: Dietary diversity was positively associated with psychological resilience among elders: A PopulationBased Study. Nutrients 2019; 11:650

27. Yuan JM, Zhang JE, Zheng MC, Bu XQ: Stigma and its influencing factors among Chinese patients with stoma. Psychooncology 2018; 27:1565-1571

28. Zheng H, Liang YM, Liu ZK: Symptoms of posttraumatic stress disorder and its related factors in the people with acquired disabilities. Chinese Mental Health Journal 2019; 33:756-761

Correspondence:

Yu Zhang, Lecturer, PhD

School of Economics \& Management, Hubei University of Arts and Science

\#296 Longzhong Road, 441053 Xiangyang, China

E-mail:hbuaszy@163.com 